\title{
Bienestar psicológico, identidad colectiva y discriminación en habitantes de barrios estigmatizados
}

Felipe E. García ${ }^{\star}$, Bernarda Castillo**, Angelina García ${ }^{\star \star *}$, Vanessa Smith-Castro ${ }^{\star * * *}$

* Doctor en Psicología. Profesor asociado, Facultad de Ciencias Sociales, Universidad Santo Tómas, Santiago, Chile. Correo electrónico: fgarcia@ santotomas.cl

** Psicóloga, Escuela de Psicología, Universidad de Las Américas, Santiago, Chile. Correo electrónico: bernardanicolecastillo@gmail.com

*** Psicóloga, Universidad de Las Américas, Santiago, Chile. Correo electrónico: angelina.garcia.ps@gmail.com

**** Doctora en Psicología Social. Profesora catedrática y directora del Instituto de Investigaciones Psicológicas, Universidad de Costa Rica, San José, Costa Rica. Correo electrónico: vanessa.smith@ ucr.ac.cr

Recibido: 30 de enero del 2017

Aprobado: 21 de agosto del 2017

Cómo citar este artículo: García, F. E., Castillo, B., García A. y Smith-Castro V. (2017). Bienestar psicológico, identidad colectiva y discriminación en habitantes de barrios estigmatizados. Pensando Psicología, 13(22), 41-50. doi: https://doi.org/10.16925/ pe.v13i22.1987

\section{Resumen}

Introducción: la discriminación social y la estigmatización de las personas por su pertenencia a un grupo determinado, en el presente caso por su lugar de residencia, es un problema relevante y que afecta el bienestar y la salud mental. Objetivo: establecer la influencia relativa de la identidad colectiva y la percepción de discriminación en el bienestar psicológico de habitantes de barrios estigmatizados. Metodología: la muestra estuvo formada por 160 personas adultas (50\% mujeres) que habitaban barrios estigmatizados de la provincia de Concepción en Chile. Los instrumentos utilizados fueron la Mental Health Continuum-Short Form (MHC-SF) de Keyes para la medición del bienestar psicológico, la Escala de Identidad Colectiva de Van Zomeren y la Escala de Experiencias de Discriminación adaptada al contexto latinoamericano por Smith-Castro. Resultados: los resultados muestran que la discriminación influye negativamente y la identidad colectiva influye positivamente en el bienestar psicológico. Conclusiones: fortalecer el sentido de pertenencia y el orgullo endogrupal podría posibilitar entornos y condiciones de vida que propicien la salud mental y formas de vida saludables.

Palabras clave: desigualdad, estigmatización territorial, discriminación territorial, exclusión social, prejuicio. 


\title{
Psychological Well-Being, Collective Identity and Discrimination in Stigmatized Neighborhoods
}

\begin{abstract}
Introduction: Social discrimination and stigmatization of individuals for belonging to a particular group, specifically for their place of residence in this case, is a major problem and affects well-being and mental health. Purpose: The objective of this study was to establish the relative influence of collective identity and the perception of discrimination on the psychological well-being of inhabitants of stigmatized neighborhoods. Method: The sample consisted of 160 adults (50\% women) living in stigmatized neighborhoods in the province of Concepción, Chile. The instruments used were Keyes's Mental Health Continuum-Short Form (MHC-SF) for the measurement of psychological well-being, the Van Zomeren's Collective Identity Scale and the Discrimination Experiences Scale adapted to the Latin American context by Smith-Castro. Results: The results show psychological well-being is negatively influenced by discrimination and positively influenced by collective identity. Conclusions: Strengthening the sense of belonging and endogroup pride could enable environments and living conditions that promote mental health and healthy lifestyles.
\end{abstract}

Keywords: inequality, territorial stigmatization, territorial discrimination, social exclusion, prejudice.

\section{Bem-estar psicológico, identidade coletiva e discriminação em moradores de bairros estigmatizados}

\section{Resumo}

Introdução: o preconceito social e a estigmatização das pessoas por seu pertencimento a um grupo determinado, neste caso, por seu lugar de residência, é um problema relevante e que afeta o bem-estar e a saúde mental. Objetivo: o propósito deste estudo foi estabelecer a influência relativa da identidade coletiva e a percepção de discriminação no bem-estar psicológico de moradores de bairros estigmatizados. Metodologia: a amostra foi formada por 160 pessoas adultas (50 \% mulheres) que moravam em bairros estigmatizados da província de Concepción, no Chile. Os instrumentos utilizados foram a Mental Health Continuum-Short Form (MHC-SF) de Keyes, para a medição do bem-estar, a Escala de Identidade Coletiva de Van Zomeren e a Escala de Experiências de Discriminação adaptada ao contexto latino-americano por Smith-Castro. Resultados: os resultados mostram que a discriminação influencia negativamente, e a identidade coletiva, positivamente no bem-estar psicológico. Conclusões: fortalecer o sentido de pertencimento e orgulho endogrupal poderia possibilitar ambientes e condições de vida que propiciassem a saúde mental e formas de vida saudáveis.

Palavras-chave: desigualdade, estigmatização territorial, discriminação territorial, exclusão social, preconceito. 


\section{Introducción}

La discriminación social es la distinción que se basa en atributos estables como: origen étnico o nacional, género, edad, discapacidad, religión, preferencias sexuales o lugar de residencia, entre otros, y que provoca perjuicio a la dignidad personal (Soberanes, 2010). Generalmente, implica una conducta de índole negativa manifestada contra los miembros de un grupo o categoría social específica solo por el hecho de ser miembros de tal grupo o categoría (Allport, 1954). A grandes rasgos, la discriminación resulta ser un ataque $u$ ofensa a la identidad, a la imagen que el individuo tiene de sí mismo.

El concepto de discriminación ha sido ampliamente utilizado en relación directa con constructos como los estereotipos y el prejuicio, a veces como conceptos intercambiables, lo cual quizá se debe a que están altamente relacionados y a que la discriminación ha sido conceptualizada como el componente comportamental del prejuicio (Brown, 1995). Sin embargo, cada uno de estos constructos hace referencia a una faceta distinta del antagonismo intergrupal y posee mecanismos particulares de manifestación, por lo que requiere de una delimitación conceptual precisa.

En esta investigación, conceptualizamos los estereotipos como las creencias consensuales sobre los atributos de los grupos sociales y sus miembros (Oakes, Haslam y Turner, 1994; Leyens, Yzerbyt y Schadron, 1994); el prejuicio como la valoración negativa y las actitudes derogatorias hacia ciertos individuos en virtud de su pertenencia a determinadas categorías sociales, étnicas o territoriales (Brown, 1995); y la discriminación, nuestro foco de interés, como aquellas conductas por medio de las cuales se niega la igualdad en el trato a una persona debido a su adscripción a una categoría social o étnica determinada (Allport, 1954; Soberanes, 2010).

La investigación ha documentado importantes efectos de la discriminación, incluyendo estados emocionales negativos como estrés y agresividad, y síntomas depresivos y de bajo bienestar como baja autoestima e insatisfacción personal, con un tamaño del efecto que va de $r=-0,13$ a $-0,23$ (Clark et al., 1999; Paradies et al., 2015; Pascoe y Richman, 2009; Schmitt et al., 2014; Smith-Castro, 2010).

Hay evidencias del efecto negativo que tiene la discriminación en la condición de vida de las personas, aunque no todos los grupos sociales responden de igual manera ante la discriminación y hay una variedad de respuestas en el interior de cada grupo social estigmatizado. Se ha establecido, por ejemplo, que la asociación entre discriminación y ajuste psicológico es más fuerte en grupos desventajados $(r=-0,23)$ que en grupos de mayor estatus y recursos $(r=-0,10$; Schmitt et al., 2014). En este artículo, analizaremos esta relación en un grupo territorial estigmatizado como delincuencial y de bajo estatus.

Los miembros de grupos socialmente estigmatizados y víctimas de discriminación pueden llegar a interiorizar los prejuicios desvalorizantes. El estigma internalizado (también conocido como autoestigmatización) lleva a aceptar creencias estigmatizantes y a aplicar los estereotipos negativos al sí mismo (Herek, Gillis y Cogan, 2009). De esta manera, es posible que los miembros de estos grupos piensen que lo que les ocurre es debido a su falta de inteligencia, a su inexperiencia y/o por su condición social, con lo que legitiman la discriminación de la cual son víctimas. Una vez que un grupo internaliza su situación, tiene menos capacidad para defenderse contra la injusticia social (Prevert, Navarro y Bogalska, 2012). La investigación reciente muestra que el estigma internalizado afecta indirectamente la calidad de vida a través de algunas dimensiones de la identidad colectiva (Fuster et al., 2014).

Por ejemplo, Smith-Castro et al. (2010) han observado que la discriminación social expone a sus víctimas ante un proceso de estigmatización que afecta negativamente su calidad de vida, sus esperanzas y sus derechos, al asociarse a sentimientos de insatisfacción personal y a emociones negativas que repercuten en su bienestar. Al respecto, el bienestar es un constructo multidimensional que en su vertiente individual está constituido por dos tradiciones, una relacionada con la felicidad (o bienestar hedónico) y otra ligada al desarrollo del potencial humano (o bienestar eudaimónico) (Ryan y Deci, 2001). Keyes, Shmotkin y Ryff (2002) han señalado el bienestar subjetivo, consistente en evaluaciones emocionales y cognitivas de lo que hacen las personas con sus vidas, como representante de la tradición hedónica (Diener, Oishi y Lucas, 2003), y el bienestar psicológico, vinculado al desarrollo del propio potencial y al buen funcionamiento social y psicológico, como representante de la tradición eudaimónica (Ryff, 1989).

Buena parte de la investigación alrededor de los efectos de la discriminación en el bienestar personal se han focalizado en la discriminación por sexo (ej. Forster et al., 2017), etnicidad o "raza" (ej. SmithCastro, 2010) y preferencia sexual (ej. Doyle y Molix, 
2014), entre otros atributos. En menor medida, se ha abordado el tema de este estudio, es decir, la discriminación social basada en el estigma territorial. Este último se refiere a la devaluación social del territorio, ciudad o barrio, la cual afecta a sus habitantes y provoca la discriminación social de estos (Kessler y Dimarco, 2013). Estos barrios son estigmatizados al ser asociados a mayores índices de pobreza, desempleo o delincuencia, entre otras condiciones sociales desfavorables (Calavrezo y Sari, 2012). Debido a que el estigma territorial constituye una marca no visible, permite implementar diferentes estrategias, como ocultar la dirección del domicilio, que hacen que su impacto en los habitantes sea diferencial.

Por ejemplo, en sectores estigmatizados como "barrios de delincuentes" en el contexto suramericano, sus habitantes vivencian su lugar de residencia como una marca de deshonra que los posiciona ante otros como personas de las cuales hay que desconfiar y como posibles delincuentes. Los habitantes del barrio afirman que difícilmente podrían conseguir un empleo diciendo de qué barrio provienen. Muchos de ellos son conscientes de que cuando su barrio es mencionado en algún medio de comunicación por algún hecho delictivo, están siendo alcanzados por ese prejuicio que afecta a todos los habitantes por igual, poniendo en práctica la estigmatización territorial (Córdova y Rincón, 2013; Kessler, 2012).

La discriminación social implica que el trato debe ser percibido como ilegítimo y debe ser interpretado como una respuesta negativa basada en la pertenencia a una categoría social determinada (Hansen y Sassenberg, 2006). La teoría de la identidad social sostiene que la conciencia de la pertenencia a un colectivo social es parte importante de la percepción del sí mismo, de allí que la interpretación de un acto injusto como resultado de la discriminación representaría una amenaza directa a una parte fundamental de la valoración personal (Tajfel y Turner, 1979; Smith-Castro, 2010).

La identidad social o colectiva es relevante para comprender mejor el impacto de la discriminación en el bienestar psicológico. La identidad colectiva es el vínculo psicológico que permite la unión de la persona con su grupo o sus grupos sociales de referencia, y para lograr este vínculo el individuo debe reunir tres características fundamentales: percibir que pertenece al grupo, asignarle una valoración a dicha pertenencia y sentir cierto afecto derivado de la consciencia de pertenecer a un grupo (Tajfel, 1981). El sentido de pertenencia al grupo es el ingrediente esencial de la identidad colectiva, ya que al mismo tiempo la persona siente que forma parte de un grupo, en donde también se diferencia de los miembros de otros grupos a los que no pertenece. La fuente de identificación del individuo es su propio grupo, al que pertenece, pero los otros grupos, externos al individuo, también juegan un papel importante, dado que cuando experimenta que es diferente a los otros, se reafirma la pertenencia a su grupo (Mercado y Hernández, 2010).

El modelo de rechazo-identificación (Schmitt y Branscombe, 2002) y algunos estudios (Schmitt et al., 2014) plantean que la discriminación fortalece la vinculación afectiva con el grupo de pertenencia y la identidad colectiva. Además, la investigación muestra que la identidad colectiva amortigua el impacto del estrés en los grupos estigmatizados. Sin embargo, el efecto de la discriminación no variaba según el tipo de grupo y el efecto amortiguador se ha encontrado solo en una minoría de estudios. A su vez, la identidad colectiva se ha asociado con bienestar o con mayor autoestima y mayor ajuste psicológico (Molero y Murillo, 2012).

Las membresías grupales y las identidades colectivas sirven como base de soporte social y amortiguan las consecuencias negativas de eventos potencialmente estresantes al proveer a los individuos de un sentido de aceptación y autovaloración, de afiliación y contacto con otros, y de ayuda concreta (Arellano, 2011). Se ha encontrado una relación positiva entre identidad étnica y bienestar personal (Smith y Silva, 2011), y entre identidad nacional y bienestar social (Espinosa y Tapia, 2001).

Así, el propósito de la presente investigación es estimar la influencia que la discriminación social y la identidad colectiva tienen en el bienestar psicológico de personas que residen en barrios estigmatizados como delincuenciales. La alta discriminación social y la baja identidad colectiva se asociarán a peor bienestar, siendo la asociación entre discriminación y bienestar más débil cuando la identidad colectiva es más fuerte.

\section{Método}

\section{Diseño}

El estudio es de tipo descriptivo y ex post facto retrospectivo con naturaleza correlacional, que consta de múltiples mediciones por medio de encuestas. La 
descripción se hace en un solo momento temporal, por lo que tiene un carácter transversal.

\section{Participantes}

Participaron 160 personas adultas en edades entre $18 \mathrm{y}$ 87 años $(M=45,60 ; \mathrm{DE}=14,66)$, de los cuales $50 \%$ son hombres y $50 \%$ son mujeres. Estas personas viven al menos desde hace un año en barrios estigmatizados de la provincia de Concepción debido a sus condiciones sociales. Estos barrios se ubican en una región de Chile que presenta uniformidad étnica. El muestreo fue no probabilístico, de carácter intencional, estratificado por sexo. Se encuestaron personas de cuatro barrios estigmatizados como de delincuentes.

\section{Instrumentos}

Percepción de discriminación. Se utilizó una versión corta de la Escala de Experiencias de Discriminación (Landrine y Klonoff, 1997; versión castellana SmithCastro, 2010). La escala consta de cinco reactivos, para los cuales se reporta la frecuencia con la que han vivido situaciones de trato injusto en una escala de Likert de cuatro puntos, de 1 (nunca) a 4 (casi siempre). Los reactivos corresponden a una dimensión denominada "discriminación manifiesta a nivel individual" (Molero et al., 2013). Ejemplos de los reactivos son: “¿Con qué frecuencia ha sido tratado(a) injustamente por empleadores, jefes, supervisores o profesores debido a que usted reside en el barrio... (p.e. barrio Emergencia)?”, y “¿Con qué frecuencia a usted le han negado cosas como un aumento, una oportunidad laboral o un mejor puesto de trabajo debido a que reside en el barrio?". Esta muestra tuvo una consistencia interna satisfactoria: $\alpha=0,70$.

Identidad colectiva. Se utilizó la escala de identidad elaborada por Leach et al. (2008; versión castellana Arancibia y Moraga, 2015). Esta versión consta de seis ítems que se responden en una escala Likert de seis puntos, de 1 (nada) a 6 (mucho). Un ejemplo de ítem es: "Sientes que tienes mucho en común con otros habitantes del barrio...”. Esta muestra tuvo una consistencia interna alta, $\alpha=0,93$.

Bienestar psicológico. Se utilizó el Mental Health Continuum-Short Form (MHC-SF) (Keyes et al., 2008; versión castellana Aragonés, Olivos y Lima, 2011). La escala consta de catorce ítems; siete de ellos miden el bienestar hedónico, que se relaciona con el placer y/o las emociones experimentadas ("Durante el último mes, ¿con qué frecuencia te sentiste feliz?”), y siete miden el bienestar eudaimónico, que corresponde al desarrollo personal ("Durante el último mes, ¿con qué frecuencia sentiste que tenías algo importante que aportar a la sociedad?"). Los reactivos se responden de 1 (nunca) a 6 (todos los días). Se utilizó el puntaje total de la escala para los análisis siguiendo el criterio de Petrillo et al. (2015), quienes señalan que la puntuación total de esta escala revela un patrón más consistente de correlaciones con otras medidas; dicho factor general en la estructura del MHC-SF fue confirmado por De Bruin y Du Plessis (2015). En este estudio, el instrumento mostró una consistencia interna muy satisfactoria de $\alpha=0,83$.

Cuestionario sociodemográfico. Se elaboró un cuestionario sociodemográfico, en el cual se registró información de: edad, nivel educacional, sector donde residen, cantidad de tiempo que han residido en el lugar, sexo, ingresos y número de integrantes del grupo familiar.

\section{Procedimiento}

Se elaboró una prueba piloto que fue aplicada a un total de cinco personas que tenían un bajo nivel de educación formal, con el propósito de evaluar la comprensión de los ítems y el tiempo de duración de la aplicación. La prueba piloto fue aplicada en un rango de 15-20 minutos y todos los participantes comprendieron cada uno de los ítems de cada escala sin ningún grado visible de dificultad.

Se contactó con informantes clave de instituciones municipales de la provincia de Concepción, quienes categorizaron los cuatro barrios más estigmatizados como de delincuentes con base en las tasas de arrestos y en la información de los medios de comunicación. Los barrios designados fueron: Emergencia de Hualpén, Libertad de Talcahuano, sector Tucapel Bajo de Concepción y Boca Sur de San Pedro de la Paz.

En los respectivos sectores, se reclutaron voluntarios en las juntas de vecinos y en algunos almacenes de barrio. No se pagó dinero por la participación. Se solicitó a cada participante firmar una carta de consentimiento informado en la que se explicaban los objetivos de la investigación y se informó respecto de la confidencialidad, la voluntariedad y el anonimato de su participación. Los cuestionarios fueron autoadministrados de manera individual en un lapso de 45 días aproximadamente. Un 30\% $(\mathrm{N}=57)$ de las personas contactadas rechazó participar, 33 por falta de tiempo, 15 por desconfianza y nueve que no eran de los barrios. 
El estudio fue aprobado en sus aspectos éticos por una comisión de la carrera de Psicología de la Universidad de Las Américas, en Concepción (Chile).

\section{Análisis de datos}

Primero, se hizo un análisis descriptivo de la población y de los instrumentos utilizados. Para probar las correlaciones entre las variables, se utilizaron correlaciones $r$ de Pearson y regresiones lineales múltiples, considerando el bienestar como variable dependiente. Para los análisis, se empleó el software estadístico sPss 20.

\section{Resultados}

La tabla 1 presenta los resultados del análisis descriptivo de las variables de estudio, con el fin de observar su comportamiento. La asimetría y la curtosis están entre los valores aconsejados para el uso de estadísticos paramétricos, aproximándose a una distribución normal (Hair et al., 1999). Las pruebas $t$ de comparaciones por sexo no revelaron diferencias significativas entre hombres y mujeres en ninguna de las medidas. Por ende, se analizaron los resultados del conjunto de la muestra.
Para examinar la hipótesis de la relación entre baja discriminación, alta identidad colectiva y bienestar, se llevaron a cabo correlaciones y una regresión múltiple. Como se esperaba, se encontró una correlación negativa entre experiencias de discriminación y bienestar psicológico $(\mathrm{r}=-0,18, \mathrm{p}=0,02)$, y una correlación positiva con identidad colectiva $(\mathrm{r}=0,49, \mathrm{p}<0,001)$. No se observa correlación significativa entre experiencias de discriminación e identidad colectiva $(r=0,01, p=0,93)$.

Se realizó un análisis de regresión múltiple, con las experiencias de discriminación y la identidad colectiva como variables predictoras y el bienestar psicológico como variable dependiente (ver tabla 2). El modelo resultó significativo, $F_{(2,157)}=29,461$, $\mathrm{p}<0,001$, con un $\mathrm{r}^{2}=0,27\left(\mathrm{R}^{2} \mathrm{adj}=0,26\right)$. En coherencia con lo esperado, la alta discriminación y la baja identidad se asocian con menor bienestar

Además, se calculó una multiplicación entre discriminación y baja identidad colectiva; sin embargo, esta interacción no tuvo una influencia significativa en el bienestar $(\beta=0,06, p=0,766)$. Debido a la ausencia de correlación de los predictores, no se contempló un análisis de mediación como complemento a este análisis general.

Tabla 1

Estadísticos descriptivos de las variables de estudio

\begin{tabular}{lcccccc}
\hline & Mín. & Máx. & Media & DE & Asimetría & Curtosis \\
\hline Experiencias de discriminación & 5 & 20 & 9,23 & 3,28 & 1,13 & 0,99 \\
Bienestar psicológico & 24 & 84 & 63,58 & 11,76 & $-0,65$ & 0,27 \\
Identidad colectiva & 6 & 42 & 25,93 & 12,38 & $-0,22$ & $-1,40$ \\
\hline
\end{tabular}

Nota. DE $=$ Desviación estándar. Elaboración propia

Tabla 2

Regresión lineal múltiple sobre bienestar psicológico $(n=160)$

\begin{tabular}{|c|c|c|c|c|c|}
\hline \multirow{2}{*}{ Variable } & \multicolumn{2}{|c|}{ Coeficientes no estandarizados } & \multirow{2}{*}{$\frac{\text { Coeficientes tipificados }}{\text { Beta }}$} & \multirow{2}{*}{$t$} & \multirow{2}{*}{ Sig. } \\
\hline & B & Error típ. & & & \\
\hline (Constante) & 57,68 & 2,91 & & 19,820 & 0,000 \\
\hline Experiencias de discriminación & $-0,67$ & 0,24 & $-0,19$ & $-2,732$ & 0,007 \\
\hline Identidad colectiva & 0,46 & 0,06 & 0,49 & 7,193 & 0,000 \\
\hline
\end{tabular}

Nota. Elaboración propia 


\section{Discusión}

Los estigmas difieren entre sí con diferentes orígenes y diversos grados; y quienes los sufren los pueden desafiar de forma enérgica o aceptar pasivamente. El estigma que concierne a este estudio tiene la peculiaridad de ser uno de carácter territorial. Al tratarse de una marca invisible (a diferencia, por ejemplo, de algún rasgo físico como el color de piel), da la libertad de utilizar estrategias personales del tipo "yo vivo aquí, pero nada tengo que ver con la gente de este barrio" o sencillamente esconder el domicilio declarando otra dirección de residencia (Kessler, 2012). El presente estudio analizó la relación entre la discriminación social percibida con base en un estigma territorial, la identidad colectiva barrial y el bienestar psicológico de personas que residen en barrios estigmatizados como de delincuentes.

Los niveles de bienestar alcanzados por los participantes son inferiores a los obtenidos en otras poblaciones (Keyes et al., 2008; Petrillo et al., 2015), probablemente por el contexto de pobreza y la misma discriminación que los afecta.

Se constató una relación inversa entre experiencias de discriminación y bienestar; la discriminación explicó el 3,2\% de la varianza del bienestar en este estudio -en coherencia con los metaanálisis sobre el tema que encontraron un tamaño del efecto entre 3 y 5\% (Paradies et al., 2015; Pascoe y Richman, 2009; Schmitt et al., 2014)-. Esto también coincide con otros estudios, en los cuales se ha encontrado que la discriminación percibida se asocia con salud mental reducida, con insatisfacción personal, con baja autoestima y con la presencia de trastornos como depresión y ansiedad (Schmitt y Branscombe, 2002). La discriminación se ha asociado a un aumento del estrés (Pascoe y Richman, 2009), lo que puede explicar su efecto negativo.

También, se encontró una relación directa entre la identidad colectiva y el bienestar. Estos resultados son consistentes con lo propuesto por autores como Molero y Murillo (2012), quienes plantean que una alta identidad grupal estaría asociada con mayor autoestima, ajuste psicológico y menor depresión. Los resultados apoyan la tesis de que la identidad social es primordial para la salud y el bienestar de las personas, ya que hay diversas formas en que una identidad colectiva puede aumentar el bienestar (Haslam et al., 2009). Sentirse parte de un grupo provee al individuo de un sentimiento de distinción, eficacia y éxito, con consecuencias psicológicas positivas y que resultan cruciales para su bienestar. Por el contrario, percibir al grupo de pertenencia con atributos considerados negativos estaría afectando el bienestar psicológico de los individuos. Así mismo, los resultados aquí coinciden con la hipótesis de que la identificación grupal puede proteger a las personas de los efectos negativos de potenciales estresores (Outten et al., 2009).

Por otra parte, no se encontró una relación estadísticamente significativa entre las experiencias de discriminación y la identidad colectiva. Algunos estudios previos han encontrado una relación inversa entre discriminación e identidad colectiva (Romero y Roberts, 2003); otros, por el contrario, han encontrado una relación positiva entre aspectos específicos de ambos constructos (Lee et al., 2015); mientras que otros estudios, como el presente, no han encontrado vinculación estadísticamente significativa entre ambas variables (Smith-Castro, 2010).

Podemos concluir que la hipótesis de que la discriminación conduce siempre a un aumento de la identidad colectiva no es cierta. Existen varias explicaciones posibles para tratar de comprender estos resultados divergentes. Por ejemplo, Lee et al. (2015) encontraron correlaciones positivas entre la discriminación percibida y el componente conductual de la identidad colectiva, no así con los componentes afectivos y cognitivos de esta última. Nuestra medida de identidad colectiva se centró en los aspectos de identificación y solidaridad con el barrio de procedencia, aspectos que pueden no verse afectados por el reconocimiento de la discriminación social. Estos resultados sugieren que la discriminación puede afectar solo ciertos aspectos de la identidad colectiva y que es necesaria mayor investigación sobre sus distintos componentes.

Otra posible explicación de esta ausencia de asociación apunta a que los individuos pueden percibir la discriminación más como un evento interpersonal que como uno intergrupal. Esto es, el efecto de la discriminación en la identidad colectiva depende a su vez de las atribuciones que hagan las personas sobre "las causas" de la discriminación. Si la discriminación no es atribuida a un aspecto central de la identidad colectiva o no es vista como un resultado de hostilidad intergrupal sino como algo personal, es posible que esta identidad no se vea afectada aun cuando las personas hayan sufrido experiencias de discriminación desagradables. Esto resulta importante en particular si se considera que nuestra medida de discriminación corresponde a una dimensión que ha sido denominada por varios autores como discriminación abierta 
o manifiesta a nivel individual (Molero et al., 2013). La posible mediación o moderación de la identidad colectiva en la relación entre percepción de discriminación y bienestar personal podría deducirse a partir del modelo de rechazo-identificación (Schmitt y Branscombe, 2002).

Finalmente, la ausencia de correlaciones significativas entre discriminación percibida e identidad colectiva podría estar vinculada con la observación de que en algunos barrios de este estudio la identidad colectiva presenta niveles muy bajos, lo que restringe el rango de valores que posibilitaría detectar las covarianzas que se han observado en otras investigaciones. Los bajos niveles de identificación pueden deberse, a su vez, a que resulta difícil desarrollar una fuerte identificación con colectivos estigmatizados (Molero et al., 2011), y en particular cuando existen posibilidades de poner en práctica estrategias individuales de desidentificación u ocultamiento de la procedencia barrial, en línea con algunas predicciones de la teoría de la identidad social (Tajfel y Turner, 1979). Futuras investigaciones podrían enfocarse a estudiar más sistemáticamente estas tres explicaciones.

Este estudio no está exento de limitaciones. Por un lado, al tratarse de un estudio transversal, no podemos hacer inferencias causales. Por otro lado, al seleccionar la muestra en forma no aleatoria, los resultados no pueden generalizarse con certeza a toda la población. De igual manera, nuestro estudio solo abordó algunos predictores del bienestar psicológico.

Futuros estudios podrían incorporar otras variables que afecten la relación entre identidad colectiva, percepción de discriminación y bienestar psicológico, tales como: los efectos de la discriminación en la autoestima y la satisfacción personal (Smith-Castro, 2005); su relación con emociones negativas como la ira, la tristeza y/o el enojo (Smith-Castro, 2010); las atribuciones percibidas de la discriminación (Quilaqueo, Merino y Saiz, 2007); y las formas de afrontamiento ante esta (Kessler y Dimarco, 2013; Córdova y Rincón, 2013). Igualmente, sería importante estudiar otros grupos que puedan estar sujetos a discriminación como adultos mayores, personas que han estado privadas de libertad, inmigrantes, personas con enfermedades de transmisión sexual o personas con discapacidades físicas y cognitivas entre otros.

\section{Conclusiones}

A pesar de sus limitaciones, el presente estudio hace un aporte para llenar un vacío en la investigación sobre estigmatización territorial. A la fecha, no conocemos otros estudios que aborden cómo la discriminación y la identidad colectiva afectan el bienestar psicológico de los habitantes de estos barrios estigmatizados específicos. De tal forma, puede ser útil para promover mayores esfuerzos de investigación sobre estos temas en estas poblaciones. Igualmente, sería de gran utilidad para el desarrollo de intervenciones comunitarias enfocadas en la promoción y prevención de la salud mental a través del fortalecimiento del sentido de pertenencia y el orgullo endogrupal, que posibiliten a su vez entornos y condiciones de vida que propicien la salud mental y permitan a las personas adoptar y mantener modos de vida saludables, con la finalidad de integrar a la población y aumentar los niveles de bienestar personal y social.

\section{Referencias}

Allport, G. (1954). The nature of prejudice. Oxford: Addison-Wesley.

Aragonés, J., Olivos, P. y Lima, M. (2011, febrero). Conectividad con la naturaleza y bienestar hedónico y eudaimónico. Comunicación presentada en el xi Congreso de Psicología Ambiental, Almería, España.

Arancibia, I. y Moraga, F. (2015). Identidad, emociones y participación política en el movimiento estudiantil chileno (seminario de grado, Universidad de Talca, Chile).

Arellano, J. (2011). Identidad social y bienestar en una comunidad rural de la costa norte del Perú (seminario de grado, Pontificia Universidad Católica del Perú, Lima).

Brown, R. (1995). Prejudice: Its social psychology. Oxford: Blackwell Publishing.

Calavrezo, O. y Sari, F. (2012). Neighborhood effects and employment outcomes: Empirical evidence from French priority neighborhoods. Urban Public Economics Review, (17), 12-55.

Clark, R., Anderson, N. B., Clark, V. R. y Williams, D. R. (1999). Racism as a stressor for African Americans: A biopsychosocial model. American Psychology, 54(10), 805-816. doi: 10.1037/0003-066X.54.10.805

Córdova, C. y Rincón, A. (2013). Trayectorias penitenciarias de pobladores de la Legua: experiencia de cárcel y retorno al territorio (seminario de grado, Universidad de Chile, Santiago de Chile).

Diener, E., Oishi, S. y Lucas, R. E. (2003). Personality, culture, and subjective well-being: Emotional and cognitive evaluations oflife. Annual Review of Psychology, 54, 403425. doi: 10.1146/annurev.psych.54.101601.145056 
Doyle, D. M. y Molix, L. (2014). Perceived discrimination and well-being in gay men: The protective role of behavioural identification. Psychology \& Sexuality, 5(2), 117-130. doi: 10.1080/19419899.2011.653689

De Bruin, G. P. y Du Plessis, G. A. (2015). Bifactor analysis of the Mental Health Continuum-Short Form (MHC-SF). Psychological Reports, 116(2), 438-446. doi: 10.2466/03.02.PR0.116k20w6

Espinosa, A. y Tapia, G. (2011). Identidad nacional como fuente de bienestar subjetivo y social. Boletín de Psicología, 102(2), 71-87.

Forster, M., Grigsby, T. J., Soto, D. W., Sussman, S. Y. y Unger, J. B. (2017). Perceived discrimination, cultural identity development, and intimate partner violence among a sample of Hispanic young adults. Cultural Diversity and Ethnic Minority Psychology, 23(4): 576-582. doi: $10.1037 / \mathrm{cdp} 0000154$

Fuster, M. J., Molero, F., Holgado, F. P. y Mayordomo, S. (2014). Enacted and internalized stigma and quality of life among people with HIV: The role of group identity. Quality of Life Research, 23(7), 1967-1975. doi: 10.1007/s11136-014-0653-4

Hair, J. F., Anderson, R. E., Tatham, R. L. y Black, W. C. (1999). Análisis multivariante (5. ${ }^{\mathrm{a}} \mathrm{ed}$.). Madrid: Prentice Hall.

Hansen, N. y Sassenberg, K. (2006). Does social identification harm of serve as a buffer? The impact of social identification on anger after experiencing social discrimination. Personality and Social Psychology Bulletin, 32(7), 983-996. doi: 10.1177/0146167206287639

Haslam, S. A., Jetten, J., Postmes, T. y Haslam, C. (2009). Social identity, health and well-being: An emerging agenda for applied psychology. Applied Psychology, 58(1), 1-23. doi: 10.1111/j.1464-0597.2008.00379.x

Herek, G. M., Gillis, J. R. y Cogan, J. C. (2009). Internalized stigma among sexual minority adults: Insights from a social psychological perspective. Journal of Counseling Psychology, 56(1), 32-43. doi: 10.1037/a0014672

Kessler, G. (2012). Las consecuencias de la estigmatización territorial: reflexiones a partir de un caso particular. Espacios en Blanco, 22, 183-186. Recuperado de http:// www.redalyc.org/articulo.oa? $\mathrm{id}=384539804007$

Kessler, G. y Dimarco, S. (2013). Jóvenes, Policía y estigmatización territorial en la periferia de Buenos Aires. Espacio Abierto, 22(2), 224-226. Recuperado de http:// www.redalyc.org/pdf/122/12226914003.pdf

Keyes, C. L., Shmotkin, D. y Ryff, C. D. (2002). Optimizing well-being: The empirical encounter of two traditions.
Journal of Personality and Social Psychology, 82(6), 1007-1022. doi: 10.1037/0022-3514.82.6.1007

Keyes, C. L., Wis-Sing, M., Potgieter, J. P., Temane, M., Kruger, A. y Van Rooy, S. (2008). Evaluation of the Mental Health Continuum-Short Form (MHC-SF) in Setswana-speaking South Africans. Clinical Psychology and Psychotherapy, 15(3), 181-192. doi: 10.1002/cpp

Landrine, H. y Klonoff, E. A. (1997). Discrimination against women: Prevalence, consequences, remedies. Londres: Sage.

Leach, C. W., Van Zomeren, M., Zebel, S., Vliek, M. L. W., Pennekamp, S. F., Doosje, B., et al. (2008). Group-level self-definition and self-investment: A hierarchical (multicomponent) model of in-group identification. Journal of Personality and Social Psychology, 95(1), 144-165. doi: 10.1037/0022-3514.95.1.144

Lee, J. P., Lee, R. M., Hu, A. W. y Kim, O. M. (2015). Ethnic identity as a moderator against discrimination for transracially and transnationally adopted Korean-American adolescents. Asian American Journal of Psychology, 6(2), 154-163. doi: 10.1037/a0038360

Leyens, J. P., Yzerbyt, V. y Schadron, G. (1994). Stereotypes and social cognition. Londres: Sage.

Mercado, A. y Hernández, A. (2010). El proceso de construcción de la identidad colectiva. Convergencia, 17(53), 229-251.

Molero, F. y Murillo, J. (2012). La satisfacción vital: su relación con el prejuicio, la identidad nacional, la autoestima y el bienestar material, en inmigrantes. Acta Colombiana de Psicología, 15, 99-101. Recuperado de http://www.scielo.org.mx/scielo.php?script=sci_arttext\&pid=S1405-14352010000200010

Molero, F., Fuster, M. J., Jetten, J. y Moriano, J. A. (2011). Living with HIV/AIDS: A psychosocial perspective on coping with prejudice and discrimination. Journal of Applied Social Psychology, 41(3), 609-626. doi: 10.1111/j.1559-1816.2011.00729.x

Molero, F., Recio, P., García-Ael, C., Fuster, M. J. y Sanjuán, P. (2013). Measuring dimensions of perceived discrimination in five stigmatized groups. Social Indicators Research, 114, 901-914. doi: 10.1007/s11205-0120179-5

Oakes, P. J., Haslam, S. A. y Turner, J. C. (1994). Stereotyping and social reality. Oxford: Blackwell Publishing.

Outten, H. R., Schmitt, M. T., García, D. M. y Branscombe, N. R. (2009). Coping options: Missing links between minority group identification and psychological 
well-being.Applied Psychology:AnInternationalReview, 58(1), 146-170. doi: 10.1111/j.1464-0597.2008.00386.x

Paradies, Y., Ben, J., Denson, N., Elias, A., Priest, N., Pieterse, A., et al. (2015). Racism as a determinant of health: A systematic review and meta-analysis. PloS one, 10(9), e0138511. doi:10.1371/journal.pone.0138511

Pascoe, E. A. y Richman, L. S. (2009). Perceived discrimination and health: A meta-analytic review. Psychological Bulletin, 135(4), 531-554. doi: 10.1037/a0016059

Petrillo, G., Capone, V., Caso, D. y Keyes, C. L. (2015). The Mental Health Continuum-Short Form (MHC-SF) as a measure of well-being in the Italian context. Social Indicators Research, 121(1), 291-312. doi: 10.1007/ s11205-014-0629-3

Prevert, A., Navarro, O. y Bogalska, E. (2012). La discriminación social desde una perspectiva psicosociológica. Revista de Psicología Universidad de Antioquia, 4(1), 7-20. Recuperado de http://pepsic.bvsalud.org/scielo.php?script=sci_arttext\&pi$\mathrm{d}=$ S2145-48922012000100002

Quilaqueo, D., Merino, M. E. y Saiz, J. L. (2007). Representación social mapuche e imaginario social no mapuche de la discriminación percibida. Atenea (Concepción): Revista de Ciencias, Artes y Letras, (496), 81-103. Recuperado de http://www.scielo.cl/scielo.php?script=sci_ arttext\&pid=S0718-04622007000200006

Ryan, R. M. y Deci, E. L. (2001). On happiness and human potentials: A review of research on hedonic and eudaimonic well-being. Annual Review of Psychology, 52(1), 141-166. doi: 10.1146/annurev.psych.52.1.141

Ryff, C. D. (1989). Happiness is everything, or is it? Explorations on the meaning of psychological well-being. Journal of Personality and Social Psychology, 57(6), 1069-1081. doi: 10.1037/0022-3514.57.6.1069

Romero, A. J. y Roberts, R. E. (2003). The impact of multiple dimensions of ethnic identity on discrimination and adolescent's self-esteem. Journal of Applied Social Psychology, 33, 2288-2305. doi: 10.1111/j.1559-1816.2003. tb01885.x

Schmitt, M. T. y Branscombe, N. R. (2002). The meaning and consequences of perceived discrimination in disadvantages and privileged social groups. En W. Stroebe y M. Hewstone (Eds.), European Review of Social Psychology (pp. 167-199). Chichester: Wiley.
Schmitt, M. T., Branscombe, N. R., Postmes, T. y García, A. (2014). The consequences of perceived discrimination for psychological well-being: A meta-analytic review. Psychological Bulletin, 140(4), 921-948. doi: 10.1037/ a0035754

Smith-Castro, V. (2005). Discriminación percibida y autoestima en jóvenes de grupos minoritarios y mayoritarios en Costa Rica. Revista Interamericana de Psicología, 39(1), 93-106. Recuperado de http://iip.ucr.ac.cr/sites/ default/files/Curriculum/Vanessa_Smith/Percepcion\%20de\%20la\%20discrimiancion\%20(2005).pdf

Smith-Castro, V. (2010). Experiencias de discriminación social de inmigrantes nicaragüenses en Costa Rica: reacciones afectivas y atribuciones causales. Revista Interamericana de Psicología, 44(2), 373-374. Recuperado de http://www.redalyc.org/articulo.oa?id=28420641018

Smith-Castro, V., Moreno, M., Román, N., Kirschman, D., Acuña, M. y Víquez, S. (2010). Discriminación social, consecuencias psicológicas y estrategias de afrontamiento en miembros de grupos sociales estigmatizados. En I. Dobles y S. Baltodano (Eds.), Dominación, compromiso y transformación social (pp. 83-104). San José: Editorial de la Universidad de Costa Rica.

Smith, T. y Silva, L. (2011). Ethnic identity and personal well-being of people of color: A meta-analysis. Journal of Counseling Psychology, 58(1), 42-60. doi: 10.1037/ a0021528

Soberanes, J. (2010). Igualdad, discriminación y tolerancia en México. Revista Mexicana de Derecho Constitucional, 22, 263-264. Recuperado de http://www.scielo. org.mx/pdf/cconst/n22/n22a9.pdf

Tajfel, H. (1981). Human groups and social categories: Studies in social psychology. Cambridge: Cambridge University Press.

Tajfel, H. y Turner, J. (1979). An integrative theory of intergroup conflict. In S. Worchel y W. G. Austin (Eds.), The social psychology of intergroup relations (pp. 33-47). Monterrey: Brooks. 